\title{
The surgical resection of dysplastic cerebellar gangliocytoma assisted by intraoperative sonography: illustrative case
}

\author{
*Yiming Li, MD, ${ }^{1}$ Jiahe Guo, MD, ${ }^{1}$ Huijie Wei, MD, ${ }^{1}$ Cuiyun Sun, MD, ${ }^{1,2}$ Yan Chai, MD, ${ }^{3}$ Xiuwei Fu, MD, ${ }^{4}$ Kai Zhang, MD, ${ }^{5}$ \\ Shengping Yu, MD, ${ }^{1}$ and Xuejun Yang, $M D^{1}$ \\ Departments of ${ }^{1}$ Neurosurgery and ${ }^{4}$ Radiology, Tianjin Medical University General Hospital, Tianjin, China; ${ }^{2}$ Department of Neuropathology, Tianjin Neurological Institute, Tianjin \\ Medical University General Hospital, Tianjin, China; ${ }^{3}$ Tianjin Neurological Institute, Tianjin Medical University General Hospital, Tianjin, China; and ${ }^{5}$ Department of Surgery, First \\ Teaching Hospital of Tianjin University of Traditional Chinese Medicine, Tianjin, China
}

\begin{abstract}
BACKGROUND Dysplastic gangliocytoma of the cerebellum (Lhermitte-Duclos disease) is an extremely rare, slow-growing hereditary mass lesion that is mainly characterized by both specific neuroradiological features and secondary hydrocephalus. Patients may present with symptoms of cerebellar mass lesion and increased intracranial pressure. As an important part of Cowden syndrome, Lhermitte-Duclos disease in adults is typically marked by PTEN gene mutation.

OBSERVATIONS The clinical management of a 31-year-old woman who suffered Lhermitte-Duclos disease was introduced in this case report. Subtotal resection was performed with the assistance of intraoperative sonography to relieve obstructive hydrocephalus, and prophylactic $\mathrm{C} 1$ laminectomy was performed to prevent possible postoperative progression of the residual lesion. Perioperative care and surgical process were clearly revealed in an accompanying video. Intraoperative sonography of Lhermitte-Duclos disease presents hyperechoic distorted thickening cortices surrounded by hypoechoic edema belt. The patient did not report any significant neurological complications or sequelae after the lesion resection.

LESSONS The authors first reported the use of intraoperative sonography in resection of adult-onset Lhermitte-Duclos disease. Hopefully, the educative case report can provide a feasible experience in the diagnosis and treatment of Lhermitte-Duclos disease.
\end{abstract}

\section{https://thejns.org/doi/abs/10.3171/CASE21451}

KEYWORDS dysplastic gangliocytoma of the cerebellum; Lhermitte-Duclos disease; Cowden syndrome; PTEN mutation; intraoperative sonography; surgical strategy

Dysplastic gangliocytoma of the cerebellum, or Lhermitte-Duclos disease, was classified as World Health Organization (WHO) grade I according to the 2016 WHO Classification of Tumors of the Central Nervous System. ${ }^{1}$ Since first described by Lhermitte and Duclos in $1920,{ }^{2}$ around 300 cases of Lhermitte-Duclos disease have been reported in the literature. ${ }^{3}$ Dysplastic cerebellar gangliocytoma may lead to mass effect in the posterior fossa and obstructive hydrocephalus, generally beginning with symptoms and signs caused by increased intracranial pressure (e.g., headache, vomiting, nausea), swollen cerebellum (e.g., vertigo, gait disturbances), and paralysis of cranial nerve. ${ }^{4}$ Small lesions may be asymptomatic or only present with relatively subtle cerebellar signs (e.g., dysmetria). ${ }^{4}$ Besides, macrocephaly has been reported to be present in approximately $20-70 \%$ of patients with Lhermitte-Duclos disease. ${ }^{5}$

Computed tomography (CT) may show a nonspecific hypoattenuating cerebellar mass in the posterior fossa, although calcification is occasionally seen. ${ }^{3,6}$ Magnetic resonance imaging (MRI) is considered a technique of choice for diagnosis of Lhermitte-Duclos disease, producing widened cerebellar folia with the characteristic striated pattern cerebellum (also described as tigroid appearance) in T2-weighted images. ${ }^{7-10}$ With both imaging techniques, hydrocephalus is demonstrated frequently. ${ }^{11}$ The corresponding images

ABBREVIATIONS CS = Cowden syndrome; CT = computed tomography; $E V D=$ external ventricular drainage; MRI = magnetic resonance imaging; WHO = World Health Organization.

INCLUDE WHEN CITING Published October 4, 2021; DOI: 10.3171/CASE21451.

SUBMITTED August 10, 2021. ACCEPTED August 18, 2021.

* Y.L., J.G., and H.W. contributed equally to this work.

(C) 2021 The authors, CC BY-NC-ND 4.0 (http://creativecommons.org/licenses/by-nc-nd/4.0/). 
in T1 are equisignal or hypointense with rare contrast enhancement diagnosis. ${ }^{4,7}$ The latest neurological imaging modalities, including positron emission tomography, diffusion-weighted imaging, and MR spectroscopy, can also aid in the diagnosis. .,12,13 $^{2}$

Whether the pathological type of Lhermitte-Duclos disease is tumor or hamartoma remains to be discussed. ${ }^{4}$ As stated by the latest WHO classification, Lhermitte-Duclos disease was categorized as the group of "neuronal and mixed neuronal-glial tumors" with WHO grade $1 .{ }^{1}$ Grossly, the cerebellar hemisphere appears as distorted enlargement, whereas no well-defined boundary could be observed between the lesion and the normal cerebellar cortex. . $^{5,14}$ Microscopic abnormality includes expansion of the granule cell layer, hypermyelination of the outer molecular layer, degeneration of Purkinje cells, and abnormal ganglia cells of different size., ${ }^{4,15}$

Lhermitte-Duclos disease is considered pathognomonic for Cowden syndrome (CS), an autosomal dominant inherited disorder, rather than a single disease. ${ }^{5,16}$ The partial loss or absence of PTEN expression (located in chromosome 10q22-23) was regarded as a histochemical feature. ${ }^{17,18}$ Germline mutations in PTEN have been identified in all cases of adult-onset Lhermitte-Duclos disease. However, even if the family history of a patient with adult onset is negative, it is highly predictive that he/she has a germline mutation in PTEN. Hence, patients with Lhermitte-Duclos disease and their families must be aware of genetic counseling and testing to find out if they have a pathogenic mutation and subsequently undergo cancer screenings regularly.

In this report, a patient with Lhermitte-Duclos disease presents with a broad spectrum of neurological conditions, unique imaging features, and detected PTEN mutation. Comprehensive management approaches, including external ventricular drainage (EVD) and subtotal lesion resection, were taken to relieve the occlusive hydrocephalus and other mass effect symptoms. Intraoperative sonographic features of adult-onset Lhermitte-Duclos disease were first reported and described. We hope this case provides practical experience with Lhermitte-Duclos disease for research, treatment, surgical strategy, and patient management.

\section{Illustrative Case}

\section{Preoperative Clinical Status}

A 31-year-old woman presented with progressive neurological decline. A year before presentation, painless blurred vision had occurred, followed by mild memory loss. During the next few months, diffuse numbness, whole-body weakness, and nonspecific neck pain had developed. Three days before admission, she was rushed to the emergency room because of unexpected nausea, severe vomiting, paroxysmal seizure, and transient loss of consciousness. MRI of the brain performed at another hospital showed a mass lesion in the posterior fossa and secondary obstructive hydrocephalus with marked dilatation of the ventricles. The patient received EVD immediately and was transferred to this hospital.

On examination, the patient could not complete the left-hand alternating movement test, finger-nose test, and left lower extremity heel-shin test, suggesting a same-side lesion in the cerebellum. The head circumference measured $60 \mathrm{~cm}$. The pupils were equal, round, and reactive to light (from 4 to $2 \mathrm{~mm}$ ). Bilateral swollen optic disc with lost physiological cup was observed. Jerky horizontal nystagmus with an increased amplitude appeared when she looked toward the left. Neither affected cranial nerve nor speech impairment was found. Further history was obtained from her mother.
The patient presented with cerebellar ataxia at a younger age and underwent left knee hemangioma resection two decades before and bilateral breast lesion resection a month earlier with histologically proved intraductal papilloma. Bedside sonographic finding was notable for a partially enlarged thyroid with nodular changes. Her parents were healthy, and there was no family history of hereditary diseases or cancer.

Radiological findings revealed characteristic appearance of LhermitteDuclos disease. Preoperative MRI presented an unclear boundary between the lesion and the normal tissue and showed hyperintensity with apparent cortical striations in the T2-weighted image and distinct striated hyperintensity in the diffusion-weighted image in the left cerebellar hemisphere. Slight enhancement was examined after the administration of gadolinium, possibly due to the vascular proliferation (Fig. 1).

\section{Perioperative Management and Lesion Resection}

The patient had received a right frontal external ventricular drain in the emergency department at another hospital before current admission. Intracranial pressure was stabilized by intermittent drainage and treatment with mannitol. Because the infection rate increases significantly after 5 days of drainage, we recatheterized in the left frontal lobe to reduce infection risk. Subsequently, bilateral suboccipital craniotomy was performed, and the dura was opened in a U-shaped fashion. The affected cerebellar hemisphere displayed significant cerebellar hypertrophy, an ectatic gyral pattern that extended into deeper layers (Fig. 2).

On intraoperative ultrasound images, the lesion showed an inhomogeneous striped zone with alternating strong-poor echo and an ambiguous outline (Fig. 3). Distorted thickening cortices, involving dilated gyri and deepening sulci, appeared nonhomogeneous and hyperechoic surrounded with hypoechoic edema belt. The gross view of the lesion showed a soft, white appearance and fair blood supply. Removing the lesion by the sulcus approach to avoid damage to the vermis cerebelli and dental nucleus, we confirmed postoperative residual cavity by using intraoperative ultrasound. Prophylactic $\mathrm{C} 1$ laminectomy was performed to allow spatial reserves for potential growth (Fig. 2). Postoperatively, radiological examination showed that occlusive hydrocephalus was relieved and symptomatic remission was obtained (Fig. 4).

\section{Histopathological Findings}

Grossly, the cerebellar hemispheres are markedly thickened and enlarged. Microscopically, widened molecular and internal granular layers of the cerebellum, filled with irregularly arranged ganglionic cells in various sizes, were consistent with the typical diagnosis of dysplastic gangliocytoma of the cerebellum, WHO grade I (hematoxylin and eosin staining). Furthermore, the immunohistochemical results verified glial fibrillary acidic protein negative and antigen $\mathrm{Kl}-67$ sparsely positive (Fig. 5).

\section{Molecular Genetics}

Detailed gene sequence of the lesion detected the PTEN mutation, c. $1026+1 \mathrm{G}>\mathrm{A}$, which was located in the intron 8. The outcome of the blood sample coincided with that of the resected lesion, suggesting germline mutation other than somatic mutation. The pathogenic gene mutation has been identified in serial cases with CS (Fig. 5). ${ }^{19}$

The process of comprehensive patient management is shown in Video 1 so that neurosurgeons can easily understand what we did. 

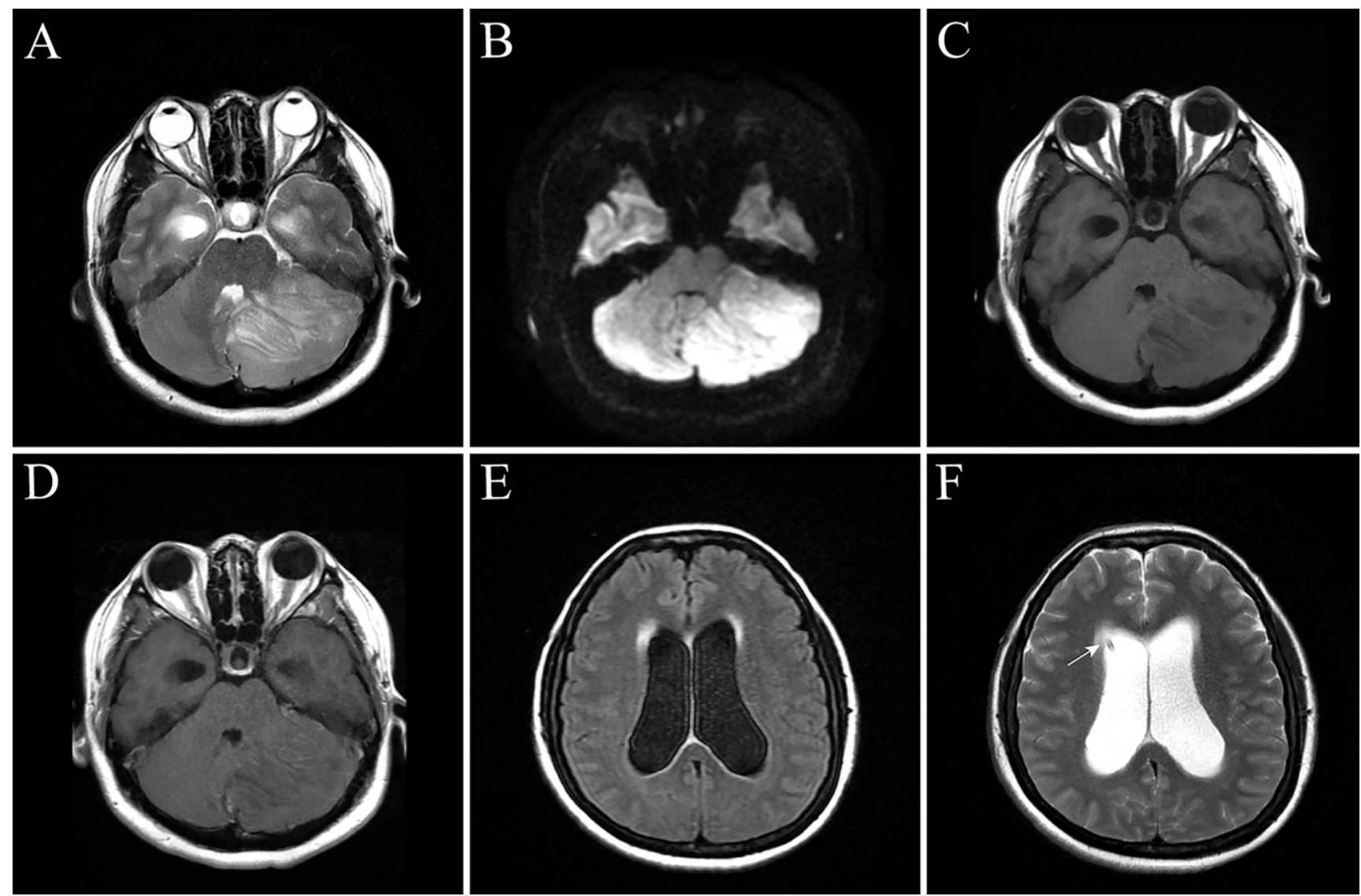

FIG. 1. Characteristic preoperative MRI in patient with Lhermitte-Duclos disease. In the left cerebellar hemisphere, T2-weighted image (A) revealed a striated feature presenting with the alternating hyper- and hypointense stripes. Diffusion-weighted image (B) shows distinct hyperintensity from the gray matter to the white matter. T1-weighted image $(\mathbf{C})$ appears indistinguishable with hypointensity, whereas slight enhancement is noted after the administration of gadolinium (D). Preoperative MRI presented an uncertain boundary between the lesion and the normal tissue. Both T2 fluid-attenuated inversion recovery (FLAIR) image (E) and T2-weighted image (F) show remarkably bilateral ventricular dilatation, suggesting secondary obstructive hydrocephalus. In T2 FLAIR, the obvious hyperintensity can be easily observed at the frontal horns of the bilateral ventricles, which indicated acute ventricular dilatation (E). The drainage tube of right frontal EVD was noted (arrow) (F).

VIDEO 1. Clip showing the diagnosis and treatment of the patient. Preoperatively, the patient reported symptoms caused by LhermitteDuclos disease and secondary obstructive hydrocephalus. Intraoperatively, we performed subtotal resection of the lesion and conducted preventive $\mathrm{C} 1$ laminectomy for possible postoperative progression of the residual lesion. Postoperative radiological examination showed that occlusive hydrocephalus was relieved, and symptomatic remission was finally obtained. We first described the ultrasonographic appearances of adult-onset Lhermitte-Duclos disease and localized the lesion by ultrasound. The extent of resection was confirmed with intraoperative ultrasound. Detailed gene sequence suggested germline mutation of PTEN. The patient did not report any significant neurological complications or sequelae after surgery. Click here to view.

\section{Discussion}

\section{Observations}

Surgical Strategy

Total resection of the mass lesion was once considered the most desirable surgical strategy for Lhermitte-Duclos disease. ${ }^{11,20}$ However, the lesion's total resection is challenging and high risk because of the slowgrowing feature and puzzling boundary with the adjacent cerebellum.

The location and volume of sacrificed cerebellar tissue are associated with loss of function. Small lesions in the vermis may cause no symptoms or deficit, but larger lesions of midline structures, such as the uvula, nodule, and flocculus, involving vestibular fibers in the cerebellum may lead to clumsiness or problems with coordination of movement, with truncal ataxia or staggering gait. ${ }^{21,22}$ Gaze-evoked or positional nystagmus occurs while the lesion damages the vestibular projections from the brainstem to the flocculonodular lobe. ${ }^{23}$ Cerebellar mutism, a transient complication with an unclear anatomical substrate, usually happens after the removal of midline tumors involving the vermis. ${ }^{24,25}$

Lhermitte-Duclos disease grows slowly and resection is often curative, with a few cases of recurrence. ${ }^{3,26}$ Jiang et al. ${ }^{27}$ reported that 18 patients with Lhermitte-Duclos disease received total or subtotal lesion resection in a single institute, and no patient had lesion recurrence during an average of 52 months of follow-up. In addition, as stated by Wang et al., ${ }^{11}$ only 1 of 9 patients who suffered Lhermitte-Duclos disease with subtotal or partial mass resection had 

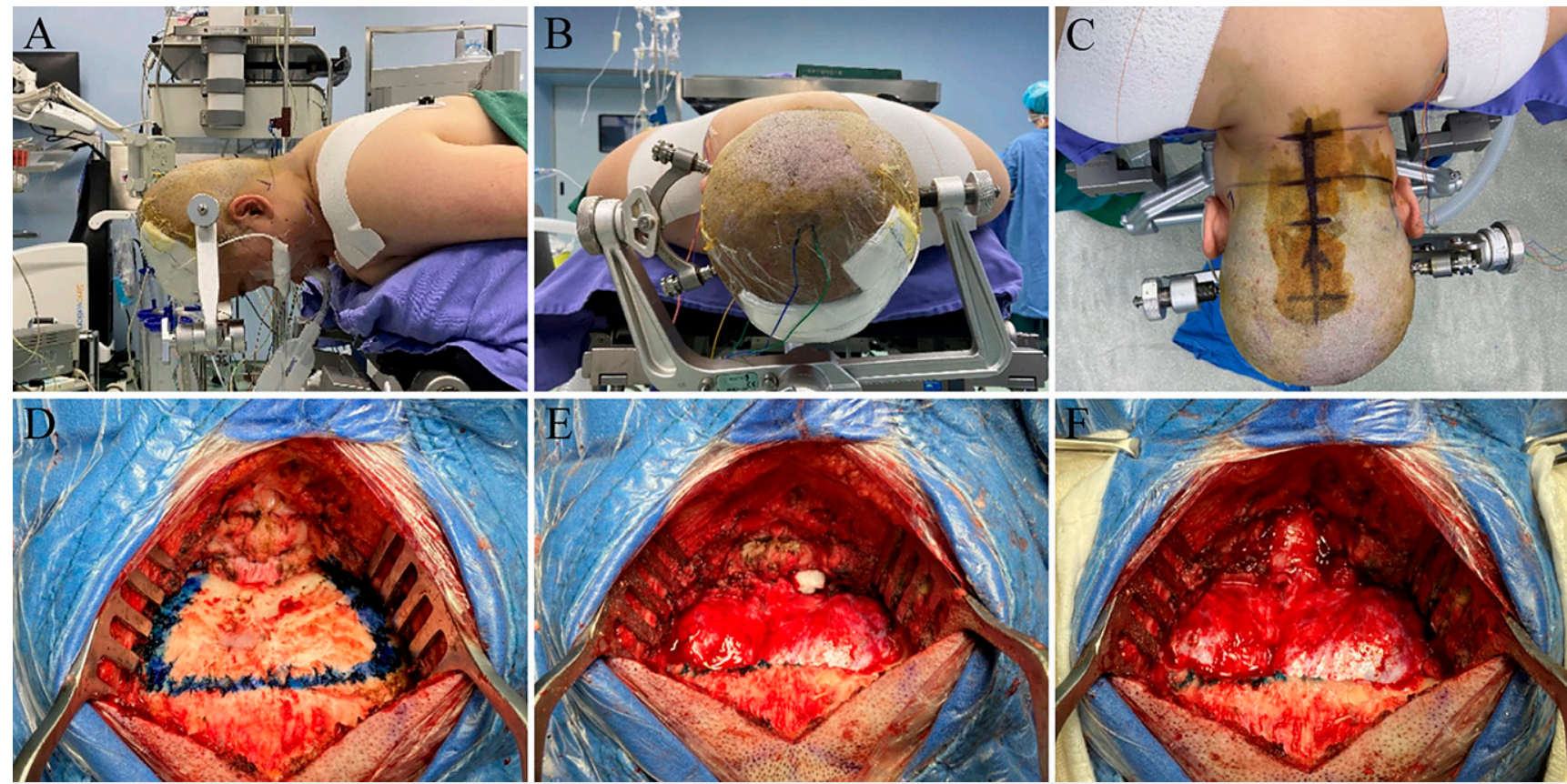

FIG. 2. Patient positioning, median suboccipital craniectomy and laminectomy of $\mathrm{C} 1$. The patient is in a prone position. The head is flexed and fixed to a Mayfield clamp, leaving a space of about $2 \mathrm{~cm}$ between the chin and the chest (A, lateral view; B, superior view). Straight skin incision started $3 \mathrm{~cm}$ above the inion and ended at the spinous process of the second cervical vertebra (C2) along the midline (C). The occipital bone and the first cervical vertebra (C2) over the posterior fossa were exposed, and the margin of suboccipital craniotomy was marked with methylene blue (D). The suboccipital craniotomy was completed $(\mathbf{E})$, followed by resection of the posterior $\mathrm{C} 1$ arch $(\mathbf{F})$.

experienced postoperative recurrence. Hence, subtotal and partial resection of Lhermitte-Duclos disease is acceptable because of its remarkably slow-growing nature. In addition, we creatively performed a laminectomy of the first cervical vertebra, a widely practiced surgical approach in the treatment of Chiari malformation and syringomyelia. ${ }^{28}$ Laminectomy of the first cervical vertebra is considered a prophylactic measure for possible postoperative progression of the residual lesion. In summary, we advocate performing both maximal safe resection and preventive $\mathrm{C} 1$ laminectomy for patients with Lhermitte-Duclos disease.
Intraoperative Ultrasound Application

Intraoperative ultrasound is regarded as an effective real-time evaluation tool in neurosurgical operations. ${ }^{29-32}$ Its uses for guidance in lesion biopsy, perioperative hemorrhage recognition, and catheter placement as well as for volume estimation and determining the integrity of tumor resection are outlined. ${ }^{31-33}$ The intraoperative sonography of adult-onset Lhermitte-Duclos disease was first recorded in the present case report. The hyperechoic stacked thickening cortices encircled by edematous hypoechoic signal were offered, consistent with the typical striated manifestation in T2-weighted
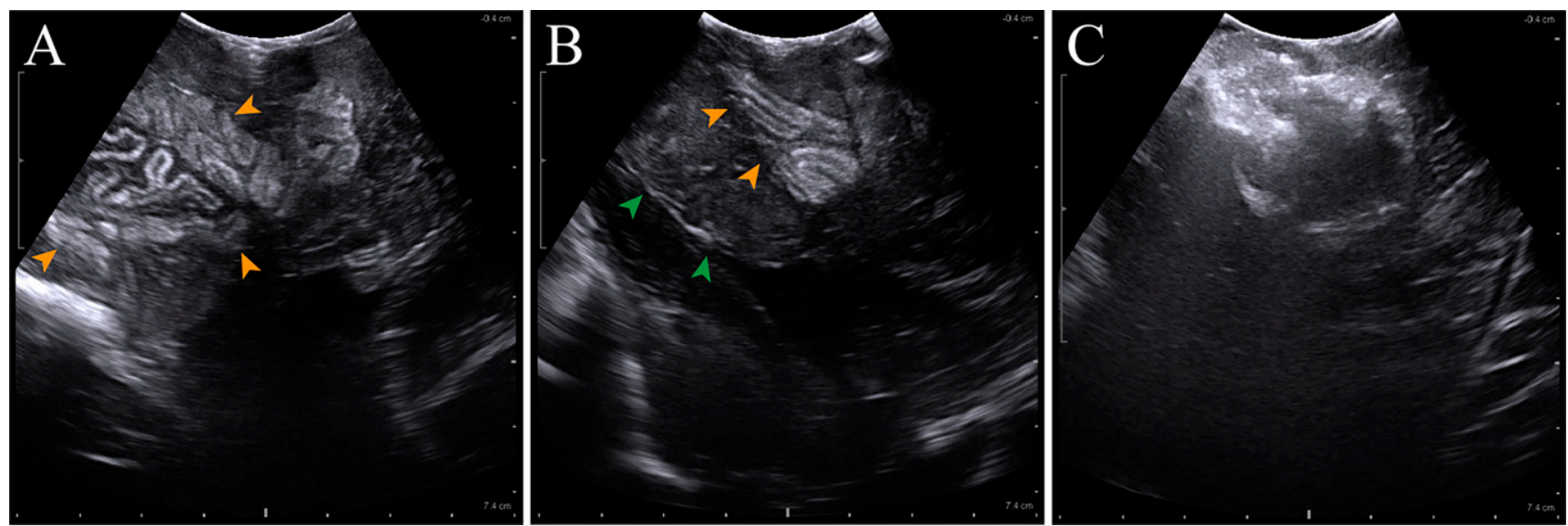

FIG. 3. Intraoperative sonography of patient with Lhermitte-Duclos disease showed an unclearly outlined lesion with alternating strong-poor striped echo (orange arrows) surrounded with hypoechoic edema belt (A, horizontal plane; $\mathbf{B}$, longitudinal plane; green arrows). The residual cavity was confirmed after lesion resection with surrounding hyperechoic hemostatic material (C, horizontal plane). 

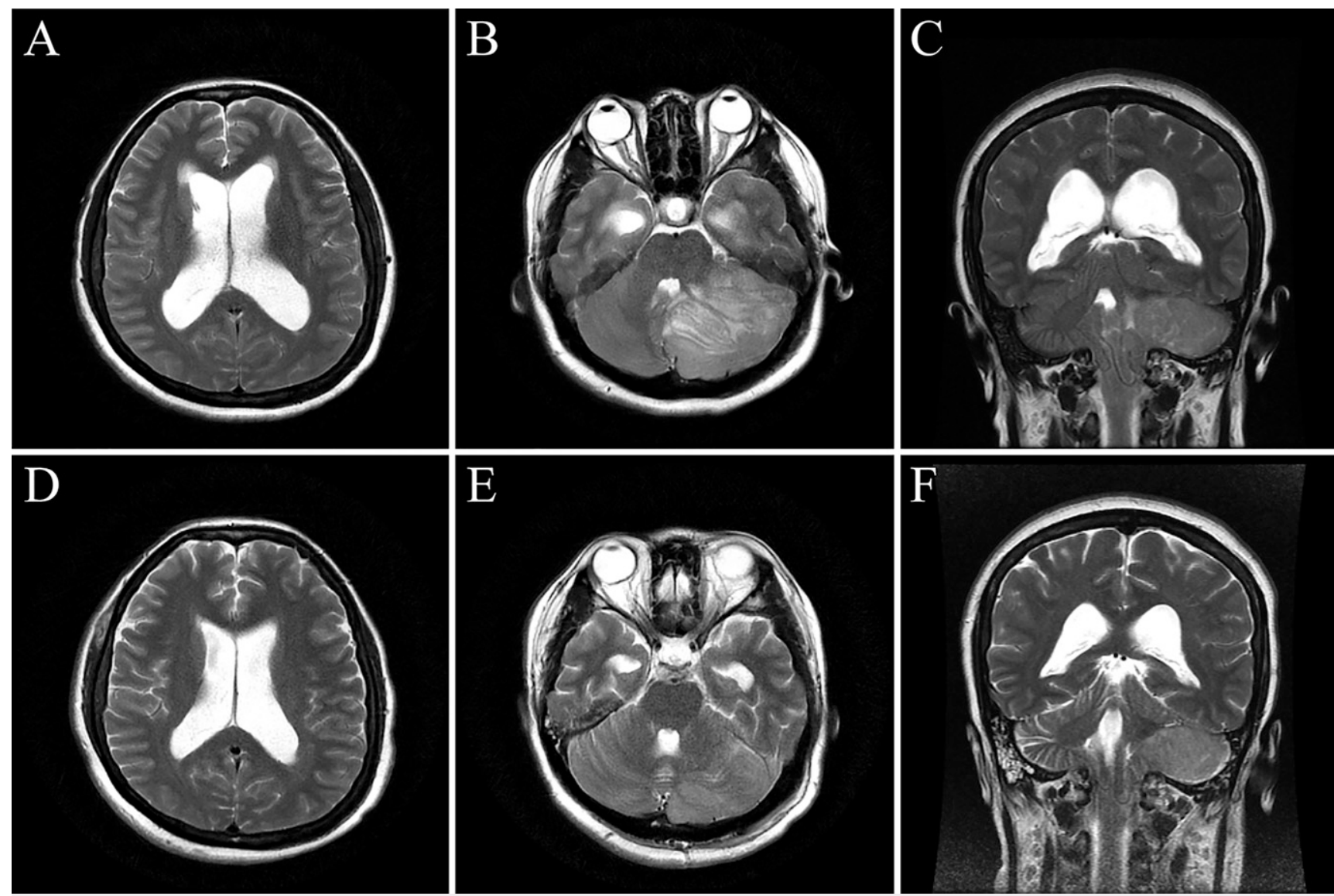

FIG. 4. Preoperative and postoperative MRI comparison. Axial T2-weighted images at the ventricular level showed that bilateral ventricular dilation and paraventricular hyperintensity $(\mathbf{A})$ were relieved after lesion resection (D). Axial T2-weighted images at the medipeduncle level showed that most of the lesion was removed (B, preoperative image; $\mathbf{E}$, postoperative image). Coronary section in T2-weighted images showed that secondary tonsillar herniation was cured (C, preoperative image; $\mathbf{F}$, postoperative image).

imaging. According to sonographic images, we determined the exact location, specified the lesion resection range, and scrutinized the blood supply and the venous sinus patency so that the lesion of Lhermitte-Duclos disease could be removed accurately. The current case demonstrated the effectiveness and practicality of intraoperative sonography during surgery for Lhermitte-Duclos disease, revealing ultrasonographic findings at the same time.

\section{Management of Patients with Lhermitte-Duclos Disease}

Generally, the diagnosis of Lhermitte-Duclos disease can be made via characteristic MRI appearance..$^{9,11,34,35}$ For accidental imaging diagnosis of patients with Lhermitte-Duclos disease, usually without any discomfort, imaging follow-up and clinical observation could be a better choice. ${ }^{9,11}$ However, because of vital tonsillar hernia, symptomatic patients with Lhermitte-Duclos disease should be treated in standard fashion. ${ }^{9}$ In this illustrative case report, the patient reported symptoms caused by cerebellar lesion and obstructive hydrocephalus and developed life-threatening cerebellar tonsillar hernia. Emergency EVD should be used for impending disaster. Considering worsening cerebellar tonsillar herniation and the infectious risk of EVD, we actively performed lesion resection and recatheterization. Furthermore, postoperative rehabilitation training is necessary to facilitate recovery.
This patient, who presented with systematic conditions consisting of Lhermitte-Duclos disease, breast tumor, and thyroid lesion, was finally diagnosed with adult-onset Lhermitte-Duclos disease associated with CS in accordance with revised PTEN hamartoma tumor syndrome clinical diagnostic criteria. ${ }^{17}$ Any individuals with multiple hamartomas or ganglioneuromas should be evaluated for CS and related conditions. Once a pathogenic PTEN gene defect is identified, other family members need genetic testing to determine presence or absence of the disease-causing mutation so that screening and surveillance can be performed. Later, the PTEN defect was proven in the patient. After confirmation of adult-onset Lhermitte-Duclos disease, the patient was probably regarded as the proband in the pedigree. Considering that her family members, especially her child, shared the same genetic information, we advised her family members on genetic testing to determine whether regular surveillance should be performed. However, her family members intended to postpone the genetic sequence because of the absence of symptoms. After her discharge, the patient still received close follow-up.

\section{Lessons}

Lhermitte-Duclos disease, a rare, noninvasive cerebellar mass composed of dysplastic ganglion cells, is a major central nervous system manifestation of CS, an autosomal dominant phacomatosis and neoplastic syndrome. This current case report introduced a patient with 


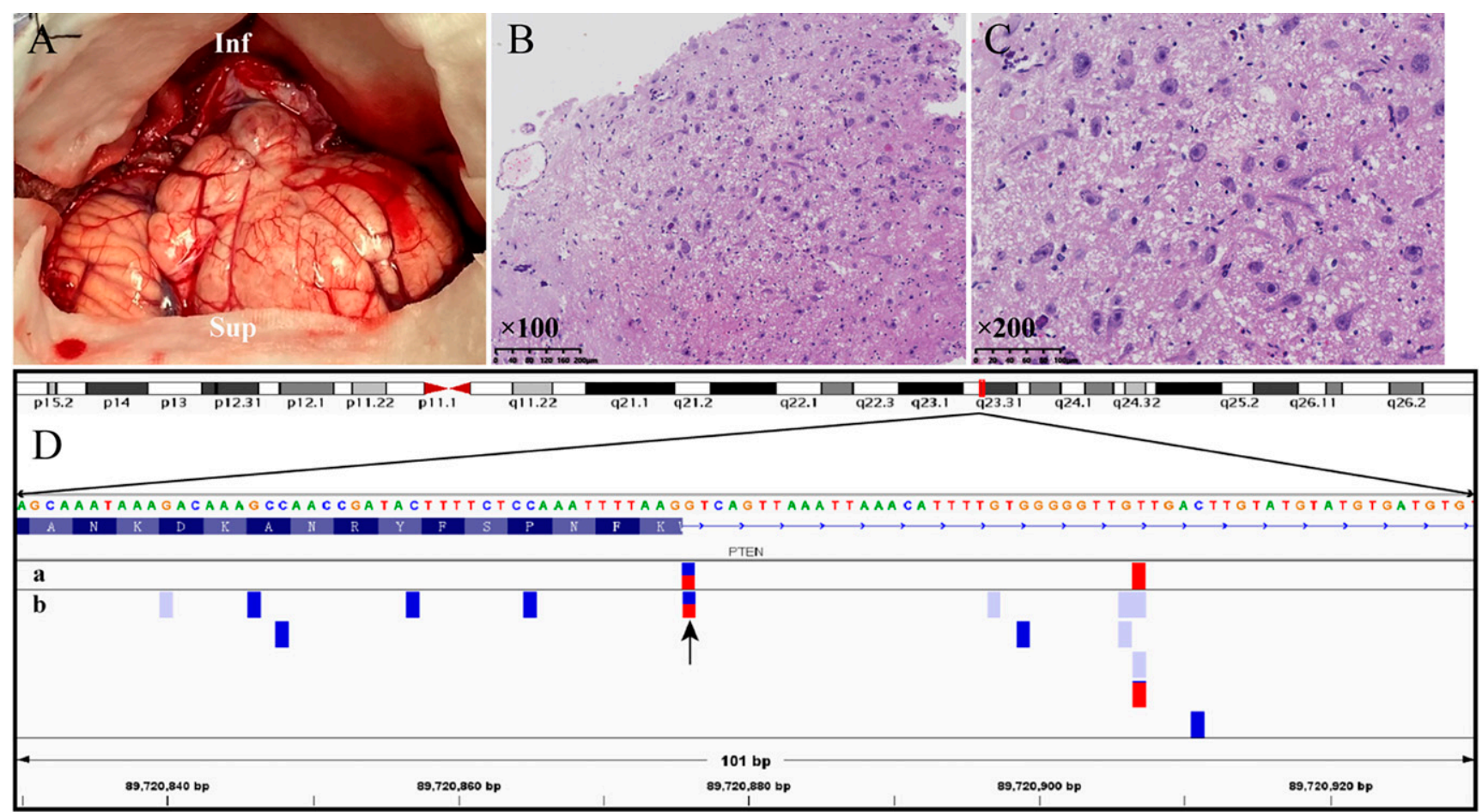

FIG. 5. Histopathology of resected lesion and germline mutation in the tumor-suppressor gene PTEN. Grossly, the surgical field presented a distorted and enlarged cerebellar hemisphere with a soft, white appearance and fair blood supply (A). In microscopic view, widened molecular and internal granular layers were filled with irregularly arranged ganglionic cells $(B$, original magnification $\times 100$; $\mathbf{C}$, original magnification $\times 200$; hematoxylin and eosin staining). Intron 8 mutation c. $1026+1 \mathrm{G}>$ A produces the mutant transcript with intron 8 retention, extending directly from exon 8 sequence (D). The same pathogenic heterozygous mutation was confirmed in both blood sample $(a)$ and tumor sample $(b)$.

Lhermitte-Duclos disease who presented with typical symptoms and signs and imaging characteristics and had an identified PTEN mutation. We first described the ultrasonographic appearance of adult-onset Lhermitte-Duclos disease and localized the mass by ultrasound. Subtotal resection and prophylactic $\mathrm{C} 1$ laminectomy are aimed at symptomatic relief and avoidance of postsurgical complications. In summary, several feasible proposals were provided through perioperative treatment, surgical strategy, and long-term management of Lhermitte-Duclos disease.

\section{Acknowledgments}

We sincerely thank fellows from the Department of Radiology, Tianjin Medical University General Hospital, for their assistance in MRI scanning. In addition, we appreciate assistance from the Department of Neuropathology, Tianjin Neurological Institute, Tianjin Medical University General Hospital. Their role in histopathological analysis could not be overlooked.

\section{References}

1. Louis DN, Perry A, Reifenberger G, et al. The 2016 World Health Organization classification of tumors of the central nervous system: a summary. Acta Neuropathol. 2016;131(6):803-820.

2. Lhermitte JDP. A diffuse cerebellar cortex ganglioneuroma. Bull Assoc Fr Etude Cancer. 1920;9:107.

3. Khandpur U, Huntoon K, Smith-Cohn M, Shaw A, Elder JB. Bilateral recurrent dysplastic cerebellar gangliocytoma (Lhermitte-Duclos disease) in Cowden syndrome: a case report and literature review. World Neurosurg. 2019;127:319-325.

4. Nowak DA, Trost HA. Lhermitte-Duclos disease (dysplastic cerebellar gangliocytoma): a malformation, hamartoma or neoplasm? Acta Neurol Scand. 2002;105(3):137-145.
5. Koch R, Scholz M, Nelen MR, Schwechheimer K, Epplen JT, Harders AG. Lhermitte-Duclos disease as a component of Cowden's syndrome. Case report and review of the literature. J Neurosurg. 1999;90(4):776-779.

6. Milbouw G, Born JD, Martin D, et al. Clinical and radiological aspects of dysplastic gangliocytoma (Lhermitte-Duclos disease): a report of two cases with review of the literature. Neurosurgery. 1988;22(1 Pt 1):124-128.

7. Dhamija R, Wood CP, Porter AB, Hu LS, Weindling SM, Hoxworth JM. Updated imaging features of dysplastic cerebellar gangliocytoma. J Comput Assist Tomogr. 2019;43(2): 277-281.

8. Meltzer CC, Smirniotopoulos JG, Jones RV. The striated cerebellum: an MR imaging sign in Lhermitte-Duclos disease (dysplastic gangliocytoma). Radiology. 1995;194(3):699-703.

9. Ma J, Jia G, Chen S, Jia W. Clinical perspective on dysplastic gangliocytoma of the cerebellum (Lhermitte-Duclos d). World Neurosurg. 2019;122:16-23.

10. Robinson S, Cohen AR. Cowden disease and Lhermitte-Duclos disease: an update. Case report and review of the literature. Neurosurg Focus. 2006;20(1):E6.

11. Wang Q, Zhang S, Cheng J, Liu W, Hui X. Lhermitte-Duclos disease: clinical study with long-term follow-up in a single institution. Clin Neurol Neurosurg. 2017;162:53-58.

12. Gaballo A, Palma M, Dicuonzo F, Carella A. Lhermitte-Duclos disease: MR diffusion and spectroscopy. Radiol Med (Torino). 2005; 110(4):378-384.

13. Thomas B, Krishnamoorthy T, Radhakrishnan VV, Kesavadas C. Advanced MR imaging in Lhermitte-Duclos disease: moving closer to pathology and pathophysiology. Neuroradiology. 2007;49(9): 733-738. 
14. Tan TC, Ho LC. Lhermitte-Duclos disease associated with Cowden syndrome. J Clin Neurosci. 2007;14(8):801-805.

15. Rimbau J, Isamat F. Dysplastic gangliocytoma of the cerebellum (Lhermitte-Duclos disease) and its relation to the multiple hamartoma syndrome (Cowden disease). J Neurooncol. 1994;18(3): 191-197.

16. Padberg GW, Schot JD, Vielvoye GJ, Bots GT, de Beer FC. Lhermitte-Duclos disease and Cowden disease: a single phakomatosis. Ann Neurol. 1991;29(5):517-523.

17. Pilarski R, Burt R, Kohlman W, Pho L, Shannon KM, Swisher E. Cowden syndrome and the PTEN hamartoma tumor syndrome: systematic review and revised diagnostic criteria. J Natl Cancer Inst. 2013;105(21):1607-1616.

18. Nelen MR, Padberg GW, Peeters EA, et al. Localization of the gene for Cowden disease to chromosome 10q22-23. Nat Genet. 1996;13(1):114-116.

19. Chen HJ, Romigh T, Sesock K, Eng C. Characterization of cryptic splicing in germline PTEN intronic variants in Cowden syndrome. Hum Mutat. 2017;38(10):1372-1377.

20. Rainov NG, Holzhausen HJ, Burkert W. Dysplastic gangliocytoma of the cerebellum (Lhermitte-Duclos disease). Clin Neurol Neurosurg. 1995;97(2):175-180.

21. Marsden JF. Cerebellar ataxia. Handb Clin Neurol. 2018;159: 261-281.

22. Morton SM, Bastian AJ. Mechanisms of cerebellar gait ataxia. Cerebellum. 2007;6(1):79-86.

23. Cho BH, Kim SH, Kim SS, Choi YJ, Lee SH. Central positional nystagmus associated with cerebellar tumors: clinical and topographical analysis. J Neurol Sci. 2017;373:147-151.

24. Catsman-Berrevoets C, Patay Z. Cerebellar mutism syndrome. Handb Clin Neurol. 2018;155:273-288.

25. Catsman-Berrevoets CE. Cerebellar mutism syndrome: cause and rehabilitation. Curr Opin Neurol. 2017;30(2):133-139.

26. Williams DW 3rd, Elster AD, Ginsberg LE, Stanton C. Recurrent Lhermitte-Duclos disease: report of two cases and association with Cowden's disease. AJNR Am J Neuroradiol. 1992;13(1):287-290.

27. Jiang T, Wang J, Du J, et al. Lhermitte-Duclos disease (dysplastic gangliocytoma of the cerebellum) and Cowden syndrome: clinical experience from a single institution with long-term follow-up. World Neurosurg. 2017;104:398-406.

28. Holly LT, Batzdorf U. Chiari malformation and syringomyelia. J Neurosurg Spine. 2019;31(5):619-628.

29. Woydt M, Vince GH, Krauss J, Krone A, Soerensen N, Roosen K. New ultrasound techniques and their application in neurosurgical intra-operative sonography. Neurol Res. 2001;23(7):697-705.
30. Sutcliffe JC. The value of intraoperative ultrasound in neurosurgery. Br J Neurosurg. 1991;5(2):169-178.

31. Sastry R, Bi WL, Pieper S, et al. Applications of ultrasound in the resection of brain tumors. J Neuroimaging. 2017;27(1):5-15.

32. Chandler WF, Knake JE, McGillicuddy JE, Lillehei KO, Silver TM. Intraoperative use of real-time ultrasonography in neurosurgery. J Neurosurg. 1982:57(2):157-163.

33. Unsgaard G, Gronningsaeter A, Ommedal S, Nagelhus Hernes TA. Brain operations guided by real-time two-dimensional ultrasound: new possibilities as a result of improved image quality. Neurosurgery. 2002;51(2):402-412.

34. Lok C, Viseux V, Avril MF, et al. Brain magnetic resonance imaging in patients with Cowden syndrome. Medicine (Baltimore). 2005;84(2):129-136.

35. Buhl R, Barth H, Hugo HH, Straube T, Mehdorn HM. Dysplastic gangliocytoma of the cerebellum: rare differential diagnosis in space occupying lesions of the posterior fossa. Acta Neurochir (Wien). 2003;145(6):509-512.

\section{Disclosures}

Xuejun Yang received funding from the National Key Research and Development Program of China (grant no. 2018YFC0115603).

\section{Author Contributions}

Conception and design: all authors. Acquisition of data: Li, Guo, Wei, Yu. Analysis and interpretation of data: Li, Guo, Wei. Drafting the article: Li, Guo, Wei, Chai. Critically revising the article: Yang, Li, Guo, Wei. Reviewed submitted version of manuscript: Yang, Li, Guo. Approved the final version of the manuscript on behalf of all authors: Yang. Administrative/technical/material support: Yang, Guo.

\section{Supplemental Information \\ Video \\ Video 1. https://vimeo.com/591052116.}

\section{Correspondence}

Xuejun Yang: Tianjin Medical University General Hospital, Tianjin, China. ydenny@126.com. 